\title{
Effect of heterogenous and homogenous air gaps on dry heat loss through the garment
}

\author{
Emel Mert • Agnes Psikuta • Marie-Ange Bueno • \\ René M Rossi
}

Received: 8 August 2014 / Revised: 30 January 2015 / Accepted: 19 February 2015 /Published online: 22 March 2015

(C) ISB 2015

\begin{abstract}
In real life conditions, the trapped air between the human body and the garment has uneven shape and vary over the body parts as a consequence of the complex geometry of the human body. However, the existing clothing models assume uniform air layer between the human body and the garment or its full contact, which may cause large error in the output of simulations. Therefore, the aim of this study was to investigate the effect of a heterogeneous vertical air gap with different configuration of folds (size and frequency) on dry heat loss using a heated cylinder (Torso). It was found that the presence of folds in the garment led to an increased heat loss from the body in comparison to a homogeneous air gap of comparable size. Interestingly, the size of folds did not have an influence on the dry heat loss. Additionally, the effect of the contact area on dry heat loss became important when exceeding a threshold of about $42 \%$. The results from this study are useful for modelling of a realistic dry heat loss through the clothing and contribute to the improvement of design of protective and active sport garments.
\end{abstract}

Keywords Air gap - Contact area - Dry heat transfer . Natural convection $\cdot$ Clothing model

E. Mert $\cdot$ A. Psikuta $(\bowtie) \cdot$ R. M. Rossi

Empa, Swiss Federal Laboratories for Materials Science and

Technology, St. Gallen, Switzerland

e-mail: agnes.psikuta@empa.ch

E. Mert $\cdot$ M.-A. Bueno

Université de Haute Alsace Haute Alsace, Laboratoire de Physique et

Mécanique Textiles, Ecole Nationale Supérieure d'Ingénieurs Sud

Alsace, Mulhouse, France

\section{Introduction}

In real life, the human body and the clothing are always in interaction with the environment, where the human body attempts to keep its core temperature constant at around $37{ }^{\circ} \mathrm{C}$ by physiological thermoregulatory processes, such as vasomotor reactions, sweating and shivering (Parsons 2003). In this humanclothing-environment system, the heat transfer from the wearer's body to the environment is affected not only by the fabric properties, such as thermal and evaporative resistance and wicking properties of the fabric (Li and Zhu 2003; Richards et al. 2008), but also by the presence of air layers, such as the trapped air underneath the garment and the boundary air layer on the garment outer surface (Gibson 1993). The thermal properties of the air layer are related to its size, which, in turn, depends on the form of the wearer's body (morphology, posture and the movement) (Li et al. 2011), the mechanical properties of the fabric (fabric stiffness and shear resulting in fabric drapability) and garment design and size (Mah and Song 2010; FrackiewiczKaczmarek 2013).

The dry heat transfer through the air within, underneath and on the garment is a complex phenomenon. The dry heat loss through a stagnant air layer is transferred by conduction, radiation and convection. The conductive heat loss rate through a stagnant air layer depends linearly on the distance between the human skin and the garment as well as the temperature gradient. The radiative heat transfer rate depends non-linearly (to the power of four according to Stefan-Boltzman law) on the temperature difference between human body and garment and the emissivity of both surfaces. The convective heat transfer underneath the clothing can occur as forced convection by forced air motion, such as wind or movement, and/or natural convection due to the buoyancy effect (Cengel and Ghajar 2007). Natural convection occurs only from a certain air gap thickness when the buoyancy force overcomes the shear and tensile forces in the fluid. Thereby, the dry heat loss in 
stagnant air gap, which is only transferred by conduction and radiation (Wissler and Havenith 2009), decreases as the air gap thickness increases. When the thickness of air gap underneath the garment is large enough i.e., few millimetres to allow air movement due to the buoyancy force, the convective heat loss prevents a linear increase of total dry heat loss (Spencer-Smith 1977; Bergman et al. 2011). In other words, it can be inferred that an air gap up to the threshold of the natural convection induces an increment in thermal insulation, whereas above that threshold, its isolative capacity is reduced by development of the natural convection.

The determination of the characteristics of natural convection inside a complex geometry such as the shape of the air layer underneath the clothing is very challenging. In previous studies, the thermal effect and the threshold of natural convection have been investigated in horizontal and vertical homogeneous planar air gaps. Gretton et al. (1998) used a wide range of fabrics with different breathability, and Cain and Farnworth (1986) used fabric samples of one to four layers ensuring that there was no air gap between layers. They indicated that the natural convection in an air gap between hot plate and fabric onsets at $13 \mathrm{~mm}$ using a horizontal sweating guarded hot plate. Although, the characteristic and the onset of natural convection in homogenous horizontal air gaps are better known, it is less relevant for real-life situations of the garment on wearer's body, which is mostly in vertical orientation with numerous folds. Spencer-Smith (1977) used different sizes of vertical homogenous air gap thickness (between 5 and $30 \mathrm{~mm}$ of air gap thickness) between vertical hot plate and fabric. It has been shown that the threshold of natural convection in vertical air gap was around $8 \mathrm{~mm}$ air gaps between smooth metal surface and smooth fabric. The effect of surface roughness, such as different skin and fabric topography and hairiness, was not investigated in this study.

The characteristic of natural convection inside a more complex geometry of vertical annuli, which was uniformly heated by an inner cylinder and had an adiabatic outer cylinder, was also investigated (Alarabi et al. 1987; Mohanty and Dubey 1996). Although the theoretical models developed in these studies were validated using experimental results for only some selected cases, they have not accounted for the friction between the air and the annulus/plate surface and possible porosity of the outer annulus. Both parameters are likely to have an effect on the development of the natural convection in the air gap with one or both limiting surfaces being textile porous materials with possible heterogeneous surface structure. The thermal effect of vertical air layers with complex geometry was not investigated until now; the existing mathematical models assume homogenous air gap or full contact between the human body and the garment (Berger and Sari 2000; Fan et al. 2000; Lotens and Havenith 1991). Although such approach simplifies the analysis of the experimental results and the mathematical simulation, it disregards the heterogeneity of the air layer thickness and its contact area at the body resulting in a heterogeneous heat loss.

The thermal conductivity rate is smaller for motionless gases such as stagnant air underneath the garment, whilst being higher for the solids such as fabric, which can be in direct contact with the human body. Thus, the direct contact between the human body and the garment increases the total dry heat loss owing to the effectively greater thermal conductivity of the porous fabric (Ismail et al. 1988). Additionally, due to this direct contact of fabric with the skin, the fabric absorbs and transfers liquid sweat away from the skin surface and distributes it over a larger area on the garment (Keiser et al. 2008; Umeno et al. 2001). Therefore, it is important to consider the spatial heterogeneity of the air gaps, which is the combination of the spatially geometrically complex air spaces and contact areas, in mathematical clothing models and clothing design. The quantification of the contact area (Psikuta et al. 2012a) and air gap thickness (Psikuta et al. 2012b; Mah and Song 2010) has been enabled by the 3D scanning technique and advanced post-processing method. However, the thermal effect on human body of a change in the amount of contact area has not been studied yet.

The thermal and physiological effects of ensembles are usually measured on thermal manikins (Anttonen et al. 2004; Oliveira et al. 2008) or using human subjects (Brode et al. 2008; Konarska et al. 2007) to obtain a realistic measure of garment performance. These methods allow the interaction of the garment with the complex geometry of the human body, and hence, formation of realistic air layer thickness. In this way, the overall thermal, in case of the manikin, and physiological, in case of the human subjects, effect of the garment together with the realistic air layers can be measured. On the other hand, the separation of the contributing heat transfer mechanisms and the factors related to the garment design and fabric properties is often limited or impossible when using such methods. This fact hinders the understanding of the underlying thermal effects, their quantification and subsequent modelling.

The aim of the present study was to systematically investigate the effect of the heterogeneous vertical air gap with different configuration of folds (size and frequency) on dry heat loss using the heated cylinder Torso. Our particular research questions in this study concerned (i) differences in heat transfer through homogenous and heterogeneous air gaps and (ii) contribution of the larger contact area to the dry heat loss due to the higher effective conductive heat transfer. The knowledge from this study shall help understanding the complexity of dry heat transfer related to garment design, contribute to an improved design of protective and functional garments and to a further development of the mathematical clothing models. 


\section{Materials and methods}

\section{Torso device}

Torso is a vertical heated sweating cylinder for measuring heat loss through fabrics and fabric composites (Keiser et al. 2008; Psikuta et al. 2013; Annaheim et al. 2014). The size of the main cylinder of Torso has been designed as the approximate size of an adult male trunk, which is $30 \mathrm{~cm}$ in diameter and $46 \mathrm{~cm}$ in height. It has two heated aluminium guards preventing lateral heat losses. Two Nickel-wire resistance temperature sensors are evenly wind on the main cylinder surface and between the sweating outlets to measure the average temperature of the main cylinder. Additionally, to distribute water evenly over the surface of Torso, tight-fitted knitted fabric (71\% polyamide, $29 \%$ spandex knitted fabric) is used to simulate human skin. These features of Torso allow the evaluation of the thermal parameters of single- and multi-layer fabric systems such as heat flux through the fabric system, thermal and evaporative resistance and water transfer properties.

\section{Air gap spacer}

In order to analyse thermal effects of different sizes and number of folds, three different air gap spacers of 10, 30 and $50 \mathrm{~mm}$ thick were used to design a setup with different sizes and distribution of folds as shown in an example in Fig. 1. Polyethylene terephthalate (PET) monofilament yarns were tied up to have additional support for forming folds. Commonly used plain weave $100 \%$ cotton woven fabric was selected as clothing. A tape measurement was placed between sticks as designed folded fabric to measure the exact length of samples to avoid stretching of the fabric. The fabric samples were cut in measured length and woven in between sticks and tightened yarns on the air gap spacer as shown in Fig. 1 and fixed in place by a few hand pick stitches.

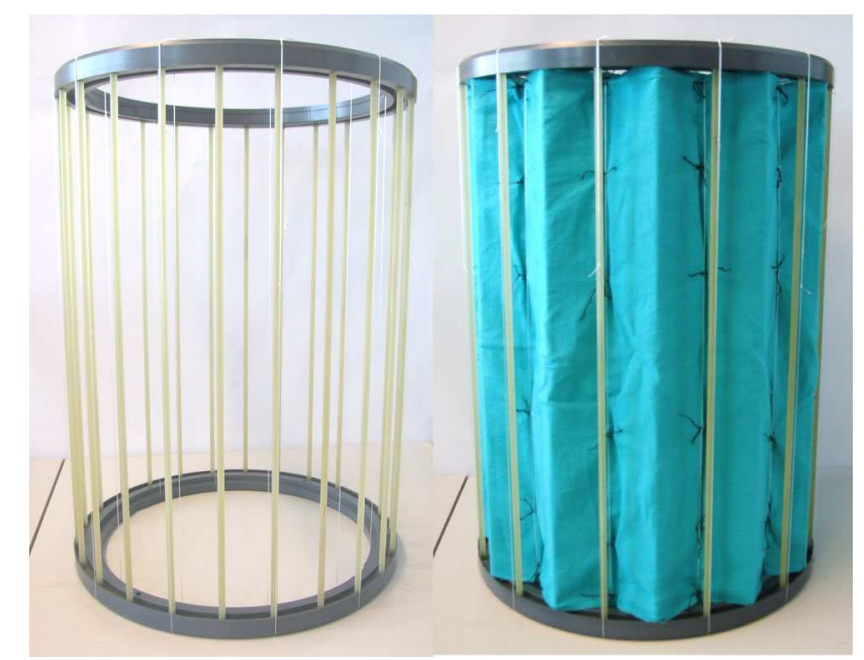

Fig. 1 Air gap spacer without and with draped fabrics
In the designs, the ratio of the width to height of folds was considered approximately three for all cases to obtain similar inclination angle of folds. Sizes of folds and the contact area were selected based on the analysis of 11 garments evaluated using accurate 3D body scanning method (Schmid 2011). The following experimental setups were prepared for this study:

- Case 1: the fabric was put tightly on Torso or on the air gap spacer in order to obtain a homogeneous air gap. The measurement using this setup was used as a reference for other cases. The parameters of the designed air gap spacer with homogenous air gap can be seen in Table 1 .

- Case 2: the fabric was woven between sticks of the air gap spacer in a zic-zac manner to obtain small, middle-sized and big folds dependent on the thickness of the spacer. The parameters of the designed air gap with different sizes of folds can be seen in Table 2 .

- Case 3: the fabric was draped on the 30-mm air gap spacer in a way to provide a various contact surfaces between folds in order to compare the effect of different sizes of contact areas resulting from different number of folds. The parameters of air gap spacer with different sizes of contact areas are shown in Table 3.

To evaluate the sample surface area, exact average air gap thickness and contact area of folds, the spacer with fabric were scanned using Artec MHT 3D scanner (Artec Group, Luxembourg), and the scans were post-processed with the dedicated surface inspection software Geomagic Qualify 2012 (Geomagic, USA) according to the method by Psikuta et al. (Psikuta et al. 2012a; Psikuta et al. 2012b). The real average air gap thickness, the contact area and the surface area of draped fabrics on air gap spacers are reported in Tables 1, 2 and 3.

\section{Measurement procedure}

The fabric samples and the skin fabric were conditioned before measurements for $24 \mathrm{~h}$ at $20 \pm 1{ }^{\circ} \mathrm{C}$ of ambient temperature and $50 \pm 5 \%$ of relative humidity in the climatic chamber. Before each measurement, the skin fabric was put on the Torso cylinder followed by the air gap spacer with draped cotton fabric. The surface temperature of Torso was kept constant at $35 \pm 0.1{ }^{\circ} \mathrm{C}$ and the ambient temperature, the relative humidity and the air velocity in the climatic chamber were kept constant as well at $20 \pm 1{ }^{\circ} \mathrm{C}, 50 \pm 5 \%$ and $0.2 \pm 0.1 \mathrm{~m} / \mathrm{s}$, respectively. The $60-\mathrm{min}$ measurements for every case were repeated three times. Dry heat loss was calculated using the following equation:

$q_{h}=\frac{H}{A_{\mathrm{T}}}$,

where $q_{h}\left(\mathrm{~W} / \mathrm{m}^{2}\right)$ is heat flux, $H(\mathrm{~W})$ is power input of the main cylinder and $A_{\mathrm{T}}\left(0.4335 \mathrm{~m}^{2}\right)$ is area of the main cylinder of Torso. 
Table 1 Parameters of set ups with homogenous air gaps (case 1)

\begin{tabular}{|c|c|c|c|c|}
\hline Setting & $\begin{array}{l}\text { Top view of the spacer } \\
\text { with woven fabric }\end{array}$ & $\begin{array}{l}\text { Sample surface } \\
\text { area }\left(\mathbf{m}^{2}\right)\end{array}$ & $\begin{array}{l}\text { Air gap thickness of } \\
\text { the spacer }(\mathrm{mm})\end{array}$ & $\begin{array}{l}\text { Contact area } \\
(\%)\end{array}$ \\
\hline $\begin{array}{l}\text { Full contact (no } \\
\text { spacer) }\end{array}$ & & 0.434 & 0 & 100 \\
\hline 10-mm Spacer & & 0.462 & 10 & 0 \\
\hline 30-mm Spacer & & 0.520 & 30 & 0 \\
\hline 50-mm Spacer & & 0.578 & 50 & 0 \\
\hline
\end{tabular}

Additionally, an infrared (IR) camera (ThermoVision ${ }^{\mathrm{TM}}$ A40M, FLIR systems, Sweden) was set up in climatic chamber to measure the surface temperature of the fabric, for the folded fabric at the foremost peak and trough. In order to record accurate IR images, the object parameters, such as emissivity (chosen 0.95 as an average fabric emissivity), atmospheric temperature, relative humidity of the air and the distance between IR camera and object, were provided for the camera software and the IR camera was acclimatized in the test environment. Images were recorded every $15 \mathrm{~min}$.

\section{Theoretical model}

The heat transfer through the homogenous air gap was simulated using a steady-state model (Wissler and Havenith 2009; Bird et al. 2001) in order to validate the experimental results of case 1 and prove credibility of the measurement method. The model consists of multiple clothing layers separated by the air gap with nodes on each interface between layers (Fig. 2). The dry heat transfer model is based on the energy balance equation (first law of thermodynamics) to be fulfilled at each node $i$ :

$q_{\mapsto i}=q_{i \mapsto}$

where $q_{\mapsto i}\left(\mathrm{~W} / \mathrm{m}^{2}\right)$ is the heat flux flowing to the node $i, q_{i \mapsto}$ $\left(\mathrm{W} / \mathrm{m}^{2}\right)$ is the heat flux flowing away from node $i$. The components of the energy balance equation are defined as follows:

$q_{\mapsto i}=\frac{T_{i-1}-T_{i}}{R_{\mapsto i}^{c}}$

$q_{i \mapsto}=\frac{T_{i}-T_{i+1}}{R_{i \mapsto}^{c}}$

where $R_{\mapsto}^{c}\left(\mathrm{~m}^{2} \mathrm{~K} / \mathrm{W}\right)$ is the thermal resistance on the left side of node $i$ and $R_{i \mapsto}^{c}\left(\mathrm{~m}^{2} \mathrm{~K} / \mathrm{W}\right)$ is the thermal resistance on the right side of node $i$. Thermal resistances of the skin fabric and the sample fabric approximate $0.013 \mathrm{~m}^{2} \mathrm{~K} / \mathrm{W}$, 
Table 2 Parameters of setups with different size of folds (case 2)

\begin{tabular}{|c|c|c|c|c|c|c|c|}
\hline Setting & $\begin{array}{l}\text { Top view of the } \\
\text { spacer with woven } \\
\text { fabric }\end{array}$ & $\begin{array}{l}\text { Ratio } \\
\text { width/height }\end{array}$ & $\begin{array}{l}\text { Height of } \\
\text { folds } \\
(\mathbf{m m})\end{array}$ & $\begin{array}{l}\text { Number of } \\
\text { folds }\end{array}$ & $\begin{array}{l}\text { Sample } \\
\text { surface } \\
\text { area }\left(\mathbf{m}^{2}\right)\end{array}$ & $\begin{array}{l}\text { Average air } \\
\text { gap thickness } \\
(\mathrm{mm})\end{array}$ & $\begin{array}{l}\text { Contact } \\
\text { area } \\
(\%)\end{array}$ \\
\hline $\begin{array}{l}\text { 10-mm } \\
\text { Spacer }\end{array}$ & & 3.93 & 10 & 24 & 0.525 & 6 & 11 \\
\hline $\begin{array}{l}\text { 30-mm } \\
\text { Spacer }\end{array}$ & & 2.62 & 30 & 12 & 0.583 & 16 & 3 \\
\hline $\begin{array}{l}\text { 50-mm } \\
\text { Spacer }\end{array}$ & & 3.14 & 50 & 6 & 0.577 & 23 & 3 \\
\hline
\end{tabular}

respectively, and the thermal resistance of air gap $R_{a}$ $\left(\mathrm{m}^{2} \mathrm{~K} / \mathrm{W}\right)$ is defined as follows:

$R_{a}=\frac{1}{\frac{k_{a}}{x_{a}}+\varepsilon \sigma\left(T_{\mapsto a}^{2}+T_{a \mapsto}^{2}\right)\left(T_{\mapsto a}+T_{a \mapsto}\right)}$

where $k_{a}(\mathrm{~W} / \mathrm{mK})$ is the thermal conductivity of air, $x_{a}(\mathrm{~m})$ is the thickness of air layer, $\varepsilon(-, \varepsilon=0.95)$ is the combined emissivity coefficient in the air layer, $\sigma\left(5.6704 e^{-8} \mathrm{~W} / \mathrm{m}^{2} \mathrm{~K}^{4}\right)$ is the Stefan Bolzman constant and $T_{\mapsto a}, T_{a \mapsto}(\mathrm{K})$ are the surface temperatures of the surfaces limiting the air layer. In this model, Wissler and Havenith (2009) considered only conductive and radiant heat transfer pathways. Finally, the thermal resistance of adjacent air layer $R_{\text {adj }}\left(\mathrm{m}^{2} \mathrm{~K} / \mathrm{W}\right)$ is defined as follows:

$R_{\text {adj }}=\frac{1}{\sqrt{a_{\text {nat }} \sqrt{T_{\text {sf }}-T_{\text {air }}}+a_{\text {frc }} v_{\text {air,eff }}+a_{\text {mix }}}+h_{\text {rad }}}$

where $h_{\text {rad }}\left(\mathrm{W} / \mathrm{m}^{2}\right)$ is radiant heat transfer coefficient, $a_{\text {nat }}$ is the regression coefficient of natural convection, $T_{\mathrm{sf}}\left({ }^{\circ} \mathrm{C}\right)$ is the surface temperature of the body, $T_{\text {air }}\left({ }^{\circ} \mathrm{C}\right)$ is the air temperature, $a_{\mathrm{frc}}$ is the regression coefficient of the forced convection, $V_{\text {air,eff }}$ is the effective air speed $(\mathrm{m} / \mathrm{s})$ and $a_{\text {mix }}$ is the regression coefficient of mixed convection. The convective heat transfer coefficient used in definition of air layer included terms for natural, forces and mixed convection regimes on the outer surface of clothing (Fiala et al. 1999; Wang 1990). The radiative heat transfer coefficient have been determined by Quintela et al. (2004), for thermal manikins and found to be constant at $4 \mathrm{~W} / \mathrm{m}^{2} \mathrm{~K}$.

The system of Eq. (1) was solved for $T_{i}$ and each node using Matlab R2011b (The MathWorks ${ }^{\circledR}$ Inc., USA). This model is limited to parallel layers with even distribution of thermal resistance and temperature over each surface. In case of folded fabric, advanced model using numerical methods is needed to calculate the effect of heterogeneous distribution of thermal resistance on dry heat loss including its implications for natural convection inside the air gap and in the adjacent air layer. Such complex model was not available for this study. Since the measurement method used for complex scenarios with folds was the same as for homogenous setups, its credibility can be also assumed for cases 2 and 3 .

\section{Statistical analysis}

The average and the standard deviation of heat flux and temperature differences between Torso and the fabric surface temperature along the foremost peak and the trough for three repetitions were calculated. In order to differentiate the heat flux through different shape and thickness of air layers beneath the fabric, one-way analysis of variance 
Table 3 Parameters of setups with different sizes of contact area (case 3)

\begin{tabular}{llllll}
\hline Setting & $\begin{array}{l}\text { Top view of the } \\
\text { Spacer with woven } \\
\text { fabric }\end{array}$ & $\begin{array}{l}\text { Ratio of } \\
\text { Width/Height }\end{array}$ & $\begin{array}{l}\text { Number } \\
\text { of folds }\end{array}$ & $\begin{array}{l}\text { Sample } \\
\text { surface area } \\
\left(\mathbf{m}^{2}\right)\end{array}$ & $\begin{array}{l}\text { Average air } \\
\text { gap thickness } \\
(\mathbf{m m})\end{array}$
\end{tabular}

(ANOVA) test for homogeneity of variance and Games Howell post hoc test were performed using statistical software PASW ${ }^{\circledR}$ Statistic Version 17.0 (IBM, SPSS Inc. Hong Kong). The results of statistical analysis were used to evaluate whether the differences in parameters, such as heat flux and surface temperature, were significantly different between various experimental cases. The confidence interval was chosen to be 0.95 .

\section{Results}

The mean heat fluxes of case 1 , case 2 and case 3 for average air gap thickness and their standard deviation are shown in Fig. 3a. Heat fluxes of vertical homogenous air gaps in the study of Spencer-Smith (Spencer-Smith 1977) and the outcome of the mathematical model described above were plotted in Fig. 3a to compare and validate the results for homogenous air gaps in our study. Figure $3 \mathrm{~b}$ presents the temperature differences between surface of Torso and the outer surface of fabrics with homogenous air gaps (case 1) and trough and peak of folded fabrics (case 2 and case 3) for average air gap thickness and their standard deviations. To evaluate the presence of natural convection, the temperature differences between the top and the bottom part of the cylinder with homogenous air gaps and their standard deviations are shown in Fig. 4.

\section{Discussion}

Our study showed that the insulation of the garment is not only a consequence of the presence of enclosed air underneath the fabric but also the shape of the air layer plays an important role. According to the presented results, the dry heat loss through the garment increased by presence of folds (Fig. 3a, case 2) in comparison to homogeneous air gaps (case 1) plausibly due to the higher local heat transfer at the trough of folds. When the fabric touches the heated surface at the trough of folds, the local thermal resistance is reduced to the sum of fabric and adjacent air layer resistances, which effectively 


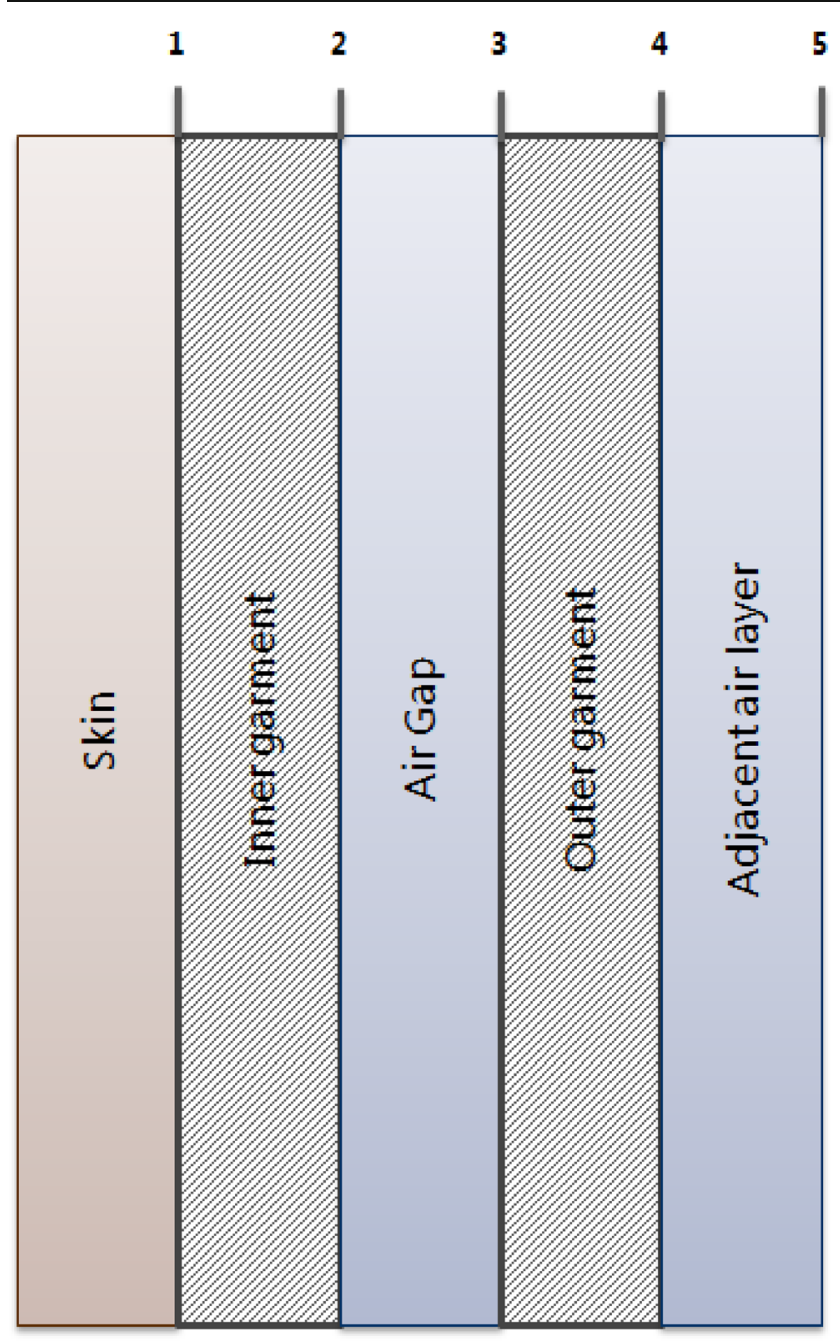

Fig. 2 Five nodes of the two-layered garment

caused an increment of the total dry heat loss. Secondly, the heat transfer phenomenon through the air gap with changing thickness is non-linear (Fig. 3a). That means that there is more excessive heat loss at low air gap in the region of the trough than reduction of that heat loss at larger air gap in the region of the peak. Thus, the difference between heat losses through a homogenous air gap and the heterogeneous one underneath the folded fabric resulted from greater energy loss around the trough (air gap smaller than the average) than energy saving around the peak (air gap larger than the average). Consequently, when the fold gets bigger, the increment of its insulation decreases and, in addition, the possible convective heat loss is more likely to occur and result in apparent decrease of the insulation. Moreover, the heat transfer through radiation gets more complex in case of folded fabric since each fabric element will exchange radiative heat not only with the hot cylinder surface (as in case of homogeneous air gap) but also with the fabric on the other side of the symmetric fold.

The dry heat loss between setups with different sizes of folds was not significantly different $(p=0.121)$, although the dry heat loss slightly increased with the increase of fold size (case 2, Fig. 3a). Since the folds were symmetric and had contact with the heated cylinder at troughs, the effect of excessive heat loss at trough region also occurred. However, it may substantially decrease when the folded fabric would be moved away from the heated surface, and hence, the differences between heat loss through troughs and peaks would be minimal (heat loss in a flatter region of the theoretical curve in Fig. 3a). Consequently, the shape of air gap layer underneath the garment is an effective factor for the increment of insulation of the garment, whilst sizes of air gap could become less important for the magnitude of radiant and conductive heat loss for larger air gaps.

Since the fabric with full contact did not have enclosed air underneath the fabric, it showed the highest dry heat loss due to higher conductivity of the fabric as a porous solid material (Fig. 3a). In case 1 with an enclosed homogenous air layer underneath the fabric, the dry heat loss started to decrease up to a certain air gap thickness and then stayed stable or even slightly increased for the largest air gap thickness tested (Fig. 3a). This result confirms the theory of increasing thermal resistance proportionally to the thickness of the stagnant air and onset of the natural convection between smooth Torso's surface and relatively smooth fabric surface at about $10 \mathrm{~mm}$ of air gap thickness. This trend matches the trend observed in the temperature difference between the Torso surface (kept constant at $35^{\circ} \mathrm{C}$ ) and the fabric outer surface measured using IR camera (Fig. 3b). The onset of the free convection could be also observed through the stratification of the air temperature in the air gap. The principle of the natural convection underneath the fabric includes the rise of warmer air (lighter due to lower density) to the top of the homogenous air gap along the heated Torso surface and sinking down of the cooler air (heavier due to higher density) along the fabric inner surface. In IR pictures in Fig. 4a, the temperature difference between the top and the bottom of homogenous air gaps was very low for 10-mm homogenous air gap and clearly higher for the larger air gaps, in which natural convection set on. On the other hand, in Fig. 4b, there is no evidence of natural convection underneath the folds in used sizes. Since the fold has narrow spaces at the heated surface and underneath the peak which may cause regional air stagnation due to friction forces, the evidence for the onset of natural convection should be sought at the sides of the folds rather than at the peak in the IR image (dashed line in Fig. 4b).

Secondly, we were able to compare trends in our results with these obtained by Spencer-Smith (1977) for vertical planar air gaps (see grey diamonds in Fig. 3a). The dry heat loss reported in this study had similar inclination and absolute values for 10 and $30-\mathrm{mm}$ air gaps as the dry heat loss from the vertical cylindrical homogenous air gaps in our study. Since the exact ambient air velocity was not reported by Spencer-Smith, the geometries were slightly different (planar versus cylindrical with relatively large radius) and it is extremely 
Fig. 3 a Heat fluxes measured for case 1,2 and 3 for average air gap thickness and these reported by Spencer-Smith (1977) for vertical homogenous air gaps (the standard deviations below 1.5 $\mathrm{W} / \mathrm{m}^{2}$ were not shown on the graph), b the temperature differences between Torso and outer surface of the fabrics in case 1 , 2 and 3 (the standard deviation below $0.6{ }^{\circ} \mathrm{C}$ were not shown on the graph)
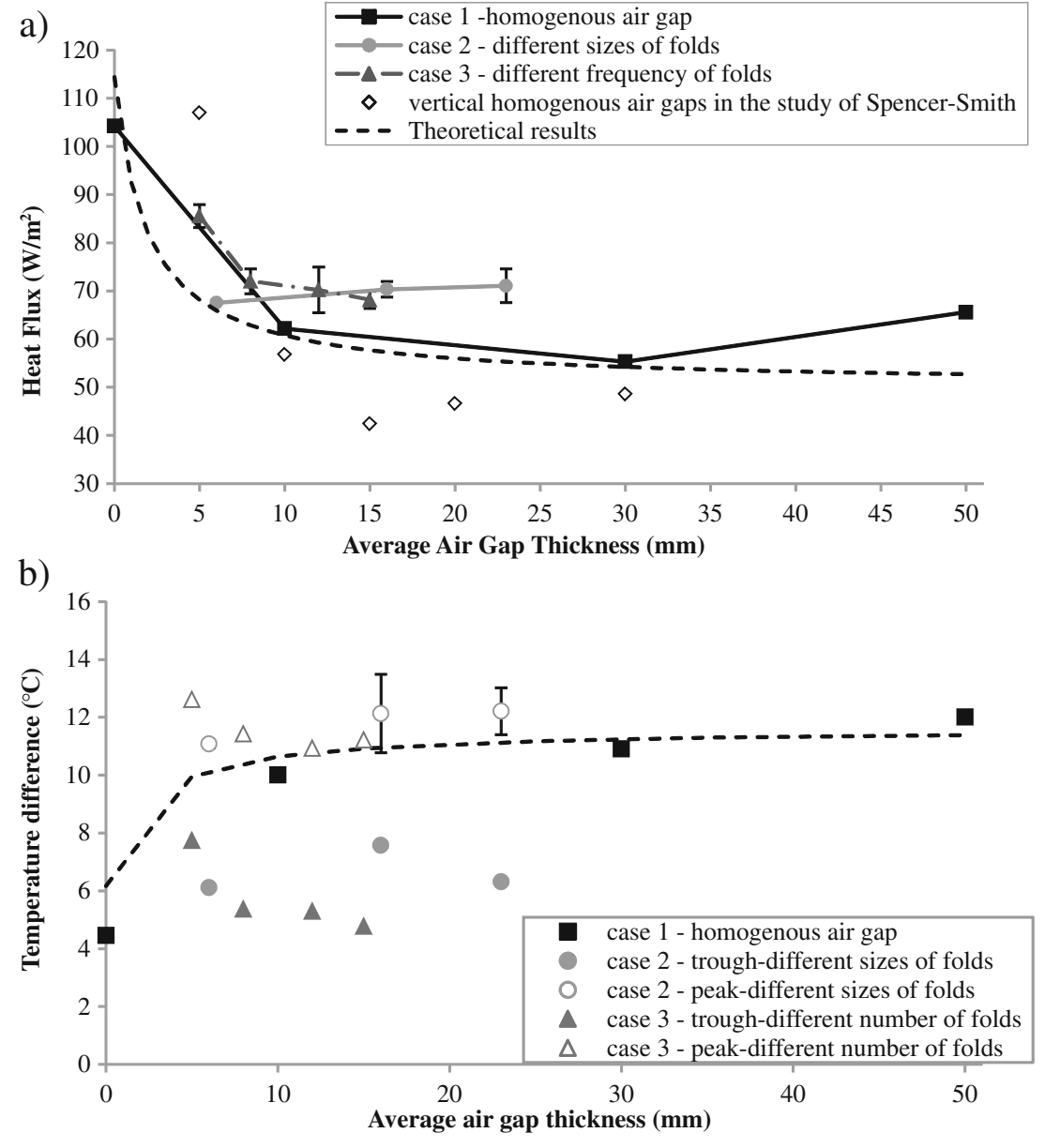

difficult to control small air gap thickness of $5 \mathrm{~mm}$ under flabby fabric, some differences between the studies were expected. Additionally, the dry heat loss through a homogeneous air gap was simulated using a mathematical model (Bird et al. 2001; Wissler and Havenith 2009) for theoretical validation. The simulation and measurement outcome had similar inclination for the heat flux and the temperature differences for 0-mm, 10-mm and 30-mm homogeneous air gaps on the vertical cylinder. Since the mathematical model does not consider natural convection, its prediction for $50-\mathrm{mm}$ air gap was expected to be less accurate due to developing free convection in the actual set up. In summary, all these facts suggest that our measurement method agrees with the theory of heat transfer through the vertical homogeneous air gap and with the experimental results obtained by other research groups. Therefore, our experimental method is a reliable practice to investigate more complex geometries of air layer underneath the fabric.

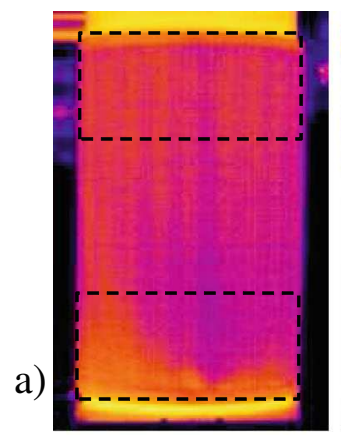

$\Delta \mathrm{T}=0.84 \pm 0.12^{\circ} \mathrm{C}$

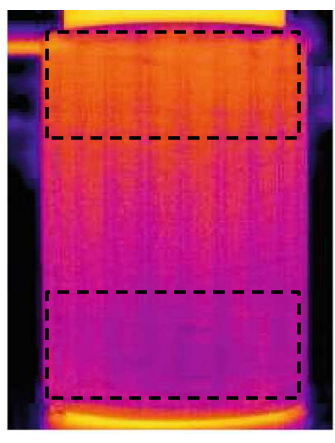

$\Delta \mathrm{T}=1.92 \quad 0.2^{\circ} \mathrm{C}$

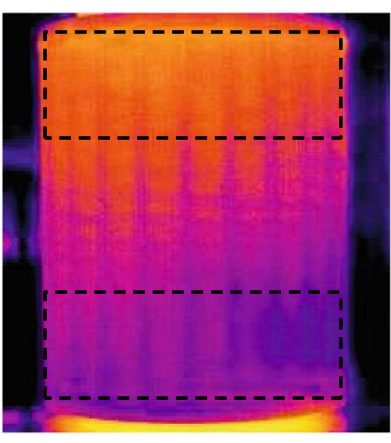

$\Delta \mathrm{T}=1.77 \quad 0.03^{\circ} \mathrm{C}$

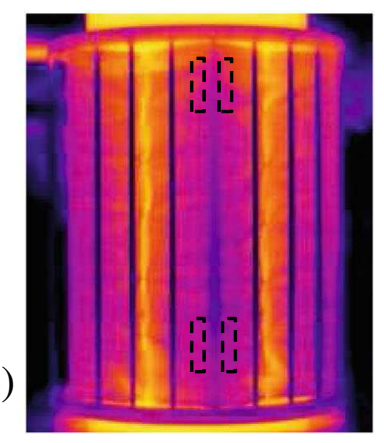

$\Delta \mathrm{T}=0.62 \quad 0.01^{\circ} \mathrm{C}$

Fig. 4 Infrared images of the fabric outer surface in a homogeneous air gap (10,30 and $50 \mathrm{~mm}$ ) configuration and $\mathbf{b}$ folded fabric (with $50 \mathrm{~mm}$ spacer) with calculated mean temperature differences between top and bottom of the cylinder from the areas marked with the dashed lines 
Interestingly, in case 3 , the effect of the contact area on dry heat transfer seems to be negligible up to a certain size of the contact area between the Torso surface and the fabric (see Fig. 3a and Table 3). The threshold falling between 42 and $67 \%$ of the surface in contact with fabric was observed due to the effective increment of heat flux via conduction. This phenomenon plausibly resulted from the increase of the conductive heat loss from the increase of the conductive heat loss from trough of folds, which functioned as a thermal bridge between heated cylinder surface and the environment. On one hand, the reason for this threshold could be the effect of the air velocity $(0.2 \pm 0.1 \mathrm{~m} / \mathrm{s})$ on the boundary adjacent air layer above the trough. Since peaks could prevent the air streams to reach the adjacent air above trough of folds in setups with denser distribution of folds, this adjacent air layer above trough could grow thicker and provided bulk of thermal resistance. However, in larger contact areas, when the distance between two peaks was large enough to lose this protective adjacent air layer due to ambient air streams, noticeable increase of dry heat loss was observed in cases with the larger contact area (distance between peaks). In such a case, the smaller sizes of folds would be expected to have smaller effect on the thickness of adjacent air layer, and hence, demonstrate increased heat loss already at lower contact area than $42 \%$. On the other hand, the heat loss curve for case 3 has a similar inclination as the theoretical model which means that there is also an effect of increasing average air gap thickness on heat loss as in homogeneous air gap. Theoretically, by adding even more folds in a series of case 3 , one would finally end up with a homogeneous air layer of $30 \mathrm{~mm}$ (last theoretical point on the curve for case 3 would be the heat loss through homogeneous air gap of $30 \mathrm{~mm}$ from case 1). This suggests that the curve of case 3 is not exactly offset to the theoretical model but rather converging to it. Therefore, it seems that both the effect of increasing average air gap thickness and the effect of decreasing direct contact and increasing air bulk on the trough sum up together in case 3 .

Additionally, the heat loss through the homogeneous and heterogeneous air layers was not directly proportional to surface area of fabrics, which is an effective factor on dry heat transfer. In case 3, the surface area increased as the thickness of air gap underneath the fabric and number of folds on setups increase (Table 3). However, the heat loss through the folded fabric decreased. Similarly, in case 1, the heat loss and the increasing surface area of homogeneous air layers did not have similar inclination. According to our results, the reason for that ineffectiveness of surface area on heat loss is the natural convection, formation of adjacent air layers on distinct geometries and complex radiative heat exchange in case of non-parallel surfaces prevailed against the effect of surface area.

According to the presented results in our study (comparison of case 1 and case 2, Fig. 3), it is clear that taking average air gap thickness and assuming homogeneous air layer underneath the garment lead to an underestimation of heat loss disregarding the spatial shape of the air layer and the contact area. Using the full contact by disregarding the air layer underneath the garment also results in overestimation of the heat transfer through the garment (comparison of case 1 and 2, Fig. 3a). However, heat and mass transfer models usually assume a uniform air layer underneath the garment either without contact area or a full contact between the skin and the fabric. The model of Lotens and Havenith (1991) is the only literature model considering the relation between the body parts and heat loss. It assumes the body as a collection of appropriately sized cylinders and the mathematical clothing model was formed based on circumferences of nude and dressed body parts. This approach offers a fast method by simplifying the computational process considering the heterogeneous distribution of air layers as an average homogenous air layer. Nevertheless, it disregards the interaction between complex geometry of human body and the garment. Since loose garments have scarce contact areas, the error for loose garments could be low. However, for regular garments with intermediate contact areas and especially for tight garments with large contact areas, this assumption may lead to large errors in calculation of heat loss. Additionally, disregarding variable contact areas, such as scarce contact areas between loose garment and the body, intermediate contact areas for regular fitted garment and large contact areas for tight fitted garment, clothing modelling may result in the underestimation of the heat loss through the garment. It is obvious that it is not realistic to take average air gap when the fabric has folds. Therefore, results from our study can be used directly in clothing modelling owing to the selection of sizes of folds in this study were based on 3D scan results of casual garments (Schmid 2011).

\section{Conclusion}

In this study, the evaluation and the comparison of the thermal effects of different sizes of folds, contact area between fabric and heated surface and the homogenous air gap thickness were performed by quantifying the heat loss and surface temperature distribution for various cases of air gap thickness and the contact area of folds. The surface temperature differences between fabrics with and without folds and the Torso were also calculated to observe the differences between the trough and the peak of folded fabrics and to estimate the natural convection underneath the fabric. Our results demonstrated that homogenous air gaps were more effective insulators than folded fabrics with the same average air gap. Additionally, although the presence of folded fabrics increased the dry heat loss through the fabric, sizes of folds were not a crucial parameter for clothing insulation. Moreover, the dry heat loss through the folded fabric became unstable and increased with 
increase of the contact area when the contact area exceeded a certain threshold of about $42 \%$ dependent on the fold size, in case of $30-\mathrm{mm}$ fold height. The knowledge gained in this study will be directly used in modelling and simulations of the dry heat transfer through the garment. Furthermore, the conclusion from this study will contribute to the improvement of clothing design for protective and active sport garments which help to balance the heat stress in microclimate between the human body and the clothing.

Acknowledgments The authors wish to thank the members of the workshop at Empa, Jörg Gschwend and Pascal Luzi, for their help and support during the study.

\section{References}

Alarabi M, Elshaarawi MAI, Khamis M (1987) Natural-convection in uniformity heated vertical annuli. Int J Heat Mass Transf 30:13811389. doi:10.1016/0017-9310(87)90170-0

Annaheim S, Psikuta A, Morrisey M (2014) A new method to assess the influence of textiles properties on human thermophysiology. Part I: thermal resistance. Int J Cloth Sc Technol in press

Anttonen H, Niskanen J, Meinander H et al (2004) Thermal manikin measurements - exact or not? Int J Occup Saf Ergon 10:291-300

Berger X, Sari H (2000) A new dynamic clothing model. Part 1: heat and mass transfer. Int J Therm Sci 39:673-683. doi:10.1016/S12900729(80)00211-6

Bergman TL, Lavine AS, Incropera FP, Dewitt DP (2011) Fundamentals of heat and mass transfer. Wiley, New York

Bird RB, Stewart WE, Lightfoot EN (2001) Transport phenomena, 2nd edn. Wiley, New York

Brode P, Havenith G, Wang XX et al (2008) Non-evaporative effects of a wet mid layer on heat transfer through protective clothing. Eur $\mathrm{J}$ Appl Physiol 104:341-349. doi:10.1007/s00421-007-0629-y

Cain B, Farnworth B (1986) Two new techniques for determining the thermal radiative properties of thin fabrics. J Build Phys 9:301-322

Cengel YA, Ghajar AJ (2007) Heat and mass transfer fundamentals and applications. McGraw-Hill, Boston

Fan JT, Luo ZX, Li Y (2000) Heat and moisture transfer with sorption and condensation in porous clothing assemblies and numerical simulation. Int J Heat Mass Transf 43:2989-3000. doi:10.1016/S00179310(99)00235-5

Fiala D, Lomas KJ, Stohrer M (1999) A computer model of human thermoregulation for a wide range of environmental conditions: the passive system. Am Physiol Soc 87:1957-1972

Frackiewicz-Kaczmarek J (2013) Determination of the air gap thickness and the contact area under wearing conditions. Doctoral Thesis, Universite de Mulhouse-Haute Alsace

Gibson PW (1993) Factors influencing steady-state heat and water-vapor transfer measurements for clothing materials. Text Res J 63:749764. doi: $10.1177 / 004051759306301208$

Gretton JC, Brook DB, Dyson HM, Harlock SC (1998) Moisture vapor transport through waterproof breathable fabrics and clothing systems under a temperature gradient. Text Res J 68:936-941. doi:10. 1177/004051759806801209

Ismail MI, Ammar ASA, El-Okeily M (1988) Heat transfer through textile fabrics: mathematical model. Appl Math Model 12:434-440
Keiser C, Becker C, Rossi RM (2008) Moisture transport and absorption in multilayer protective clothing fabrics. Text Res J 78:604-613. doi: $10.1177 / 0040517507081309$

Konarska M, Soltynski K, Sudol-Szopinska I, Chojnacka A (2007) Comparative evaluation of clothing thermal insulation measured on a thermal manikin and on volunteers. Fibers Text East Eur 15:73-79

Li Y, Zhu QY (2003) A model of coupled liquid moisture and heat transfer in porous textiles with consideration of gravity. Numer Heat Transf A Appl 43:501-523.doi:10.1080/ 10407780390122871

Li X, Wang Y, Lu Y (2011) Effects of body postures on clothing air gap in protective clothing. J Fiber Bioeng Inf 4:277-283

Lotens WA, Havenith G (1991) Calculation of clothing insulation and vapor resistance. Ergonomics 34:233-254. doi:10.1080/ 00140139108967309

Mah T, Song G (2010) Investigation of the contribution of garment design to thermal protection. Part 1: characterizing air gaps using threedimensional body scanning for women's protective clothing. Text Res J 80(13):1317-1329

Mohanty AK, Dubey MR (1996) Buoyancy induced flow and heat transfer through a vertical annulus. Int J Heat Mass Transf 39:20872093. doi:10.1016/0017-9310(95)00280-4

Oliveira AVM, Gaspar AR, Quintela DA (2008) Measurements of clothing insulation with a thermal manikin operating under the thermal comfort regulation mode: comparative analysis of the calculation methods. Eur J Appl Physiol 104:679-688. doi:10.1007/s00421008-0824-5

Parsons K (2003) Human thermal environments: the effect of hot, moderate and cold environments on human health, comfort and performance, 2nd edn. Taylor \& Francis, London

Psikuta A, Frackiewicz-Kaczmarek J, Frydrych I, Rossi R (2012a) Quantitative evaluation of air gap thickness and contact area between body and garment. Text Res J 82:1405-1413. doi:10.1177/ 0040517512436823

Psikuta A, Wang LC, Rossi RM (2013) Prediction of the physiological response of humans wearing protective clothing using a thermophysiological human simulator. J Occup Environ Hyg 10: 222-232. doi:10.1080/15459624.2013.766562

Quintela D, Gaspar A, Borges C (2004) Analysis of sensible heat exchanges from thermal manikin. Eur J Appl Physiol 92:663-668. doi:10.1007/s00421-004-1132-3

Richards MGM, Rossi R, Meinander H, Broede P, Candas V, den Hartog E, Holmer I, Nocker W, Havenith G (2008) Dry and wet heat transfer through clothing dependent on the clothing properties under cold conditions. Int J Occup Saf Ergon 14: 69-76

Schmid M (2011) Evaluation of dry heat transfer through creased fabric. Master thesis, ETH Zurich

Spencer-Smith JL (1977) The physical basis of clothing comfort part 2: heat transfer through dry clothing assemblies. Cloth Res J 5:3-17

Umeno T, Hokoi S, Takada S (2001) Prediction of skin and clothing temperatures under thermal transient considering moisture accumulation in clothing. ASHRAE Trans 107:71-81

Wang XL (1990) Free convective heat transfer coefficients of a heated full-scale manikin. Climate Buildings 1:17-31

Wissler EH, Havenith G (2009) A simple theoretical model of heat and moisture transport in multi-layer garments in cool ambient air. Eur J Appl Physiol 105:797-808. doi:10.1007/s00421008-0966-5 